\title{
Development of Noncontact Thermal Rewritable System using Laser Light
}

\author{
Tatsuo Wada*, Takashi Fujihara, Tsuyoshi Muto and Jie Gu \\ Supramolecular Science Lab., RIKEN (The Institute of Physical and Chemical Research), \\ Hirosawa 2-1, Wako, Saitama 351-0198, Japan, \\ tatsuow@postman.riken.jp
}

Keywords: rewritable, laser writing, NIR-absorbing dye, noncontact writing

\section{Introduction}

The leuco dye-based rewritable material is far superior in visibility for presenting blue or black color images on a white background by melting color development. By heating a recording layer containing the leuco dye and a developer, the separated crystals are melted and mixed to develop color (melting color development), and thus the characters and images appear. Upon abrupt cooling, it becomes amorphous and fixes the colored state. Upon heating and gradual cooling, it returns to the former phase separated state (crystal) and loses color. By controlling the coloring and decoloring of the leuco dye used in heat sensitive papers in this manner, reversible heat-sensitive (thermal rewritable) characteristics are achieved. [1]

The results of repeated printing and erasing experiments show that the rewritable material is stable even after 500 repetitions, and this repeated writing and erasing helps conserve resources and energy. The material is currently attracting attention as a third media to replace the papers and displays as a digital paper. As it allows information in the IT world to be grasped as an entity like newspapers or books, it is expected to make information-oriented society more human-friendly. The material is already widely used for shopping point cards, members' cards, commuter passes, and ID cards of various institutes. To make effective use of RFID (radio frequency identification, wireless IC) tags, it is necessary to be able to write in the rewritable material without contact. Meanwhile, to address environmental problems and personal information issues, reuse (recycling system) of the RFID tags must be considered, and so the rewritable material also needs to be recyclable. In this paper, we discuss the development of noncontact thermal rewritable system.

\section{Experimental}

The $\mathrm{Cu}$ film was prepared on thermal rewritable papers by vacuum deposition. The light-to-heat conversion layer was prepared on the same substrate by spin coating [2]. UV-vis-NIR absorption spectra of soluble phthalocyanine or the light-to-heat conversion thin films were measured by a Shimadzu UV 3100PC spectrometer. Noncontact writing was performed by using a Chromic XY-1S laser writing system with a YAG laser.

\section{Results and Discussion}

For imparting thermal energy to the rewritable material in a noncontact manner, writing by a laser beam needs to be combined with a light-to-heat conversion material (near-infrared absorbing material) that efficiently converts the laser beam to thermal energy. In summary, the light-to-heat conversion function to the rewritable material, writing, and erasing by a laser beam are necessary for the development of the noncontact thermal rewritable system.

Various laser beams are used as light sources, such as carbon dioxide gas laser $(10.64 \mu \mathrm{m})$ of near-infrared YAG laser $(1064 \mathrm{~nm})$, and semiconductor laser $(\sim 800 \mathrm{~nm})$. A noncontact 
optical writing system by a carbon dioxide gas laser as the direct heat source has been commercialized, but problems include mechanical brittleness, difficulty of handling the gas laser, and high cost. In contrast, the YAG laser and semiconductor laser are solid lasers, having merits such as mechanical stiffness and ease of handling. The YAG laser in particular can be made a compact light source by using the semiconductor laser as an excitation light source.

Conjugated compounds having conjugated $\pi$-electrons have absorption edges extended to the long wavelength region, and can be used as a near-infrared absorbing material in which the absorption band is in the oscillation regions of the optical information communication wavelength or semiconductor laser. Polyacetylene is a typical extended conjugated compound: it has a band gap of about $1.4 \mathrm{eV}$, and has absorption band across the entire visible region.[3] Polyaniline, known as aniline black, has large absorption in the near-infrared region due to acid doping, and also has peculiar absorption in the visible region.[4] Phthalocyanine dyes have absorption from $800 \mathrm{~nm}$ to the near-infrared region, according to its aggregates state, and is used as a photoreceptor for electrophotography or optical device recording dyes (Fig. 1). [2,5]

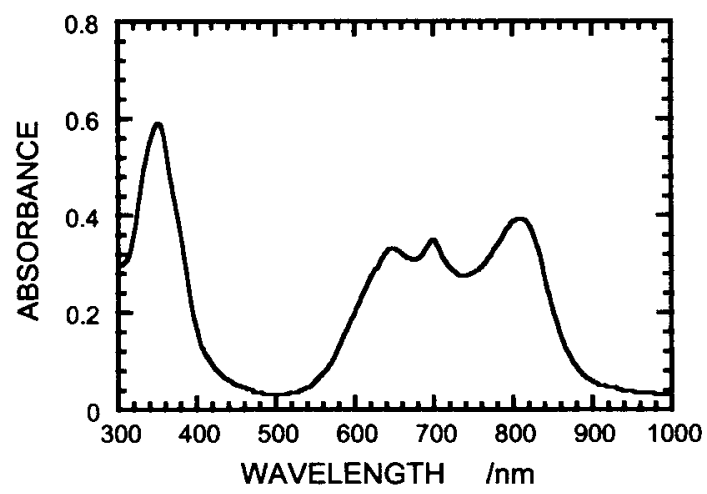

Fig. 1. Electronic absorption spectrum of soluble phthalocyanine film.

However, these compounds have absorption in the visible region, and have problems for giving visibility to RFID tags. In general, near-infrared absorbing dyes are unstable, and metallic thin films are being studied as a light-to-heat conversion material aiming at thermal stability. A copper vacuum-deposited film can be used to control the near-infrared absorption by varying its film thickness. A film thickness of $50 \AA$ allows drawing with the YAG laser. However, a plasmon resonance peculiar to copper causes the images to be colored green shown in Fig. 2 . Thus, since the conventional near-infrared absorbing materials have absorption also in the visible region, even if they are combined with decoloring or coloring rewritable papers, a high contrast cannot be obtained. To enhance the visibility for machine reading of code information, a transparent near-infrared dye having no absorption in the visible region $(400-700 \mathrm{~nm})$ needs to be developed.

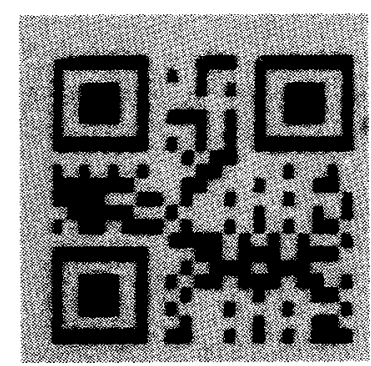

Fig. 2. QR code ${ }^{\circledR}$ drawn in the $\mathrm{Cu}$-deposited thermal rewritable paper.

The novel near-infrared dyes have a sharp absorption in the $750 \mathrm{~nm}$ region. By adjusting the concentration to the matrix, the near-infrared dyes associate by self-organization, and its absorption band shifts to a long wavelength region as shown in Fig. 3. The absorption can be adjusted in the long wavelength side of 1000 $\mathrm{nm}$ or higher. As the sensitivity in both wavelength regions of the YAG laser and semiconductor laser can be adjusted, these materials can be an efficient light-to-heat conversion material of the near-infrared laser for rewritable papers. The material has no absorption in the visible region, contributes greatly to visibility, and is expected to be applicable to various fields. This colorless transparent light-to-heat conversion material is sufficiently stable against heat, oxygen and water, and although there is some variation in absorbance caused by UV irradiation, it is negligible in practice.

An example of a $\mathrm{QR}$ code ${ }^{(}$(two-dimensional code) drawn in an optically addressible rewritable medium (thermal rewritable paper with the newly developed light-to-heat conversion material) using a YAG laser is shown in Fig. 4. In a 


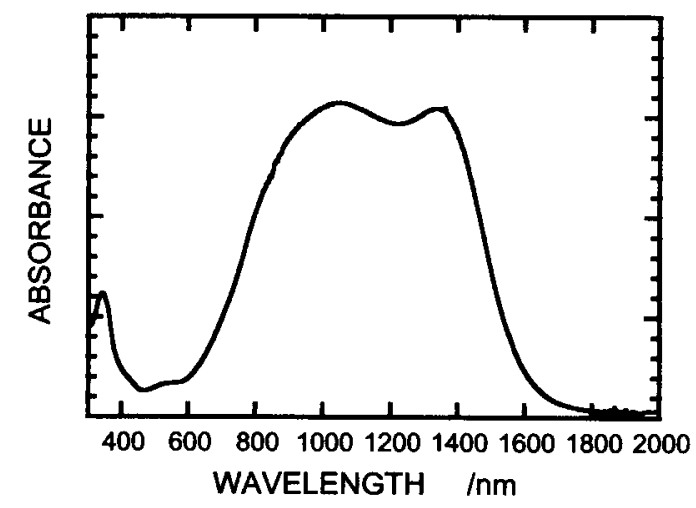

Fig. 3. Electronic absorption spectrum of light-to-heat conversion material.

printing experiment using a noncontact optical write/read instrument manufactured by Chromic Inc., printing on leuco-based rewritable papers was possible at a writing speed of $250 \mathrm{~mm} / \mathrm{s}$, at a distance of $20 \mathrm{~cm}$ or more from the light source. The printing of $\mathrm{QR}$ code ${ }^{\circledast}$ was of high quality, and proved to be automatically readable.

It has been verified that the noncontact thermal rewritable system by a laser beam greatly increases the durability of the rewritable papers, compared to the contact writing system by a thermal head with repeated writing or erasing. The rewritable systems would help conserve resources, and the technology is expected to broaden the fields of application of RFID tags.

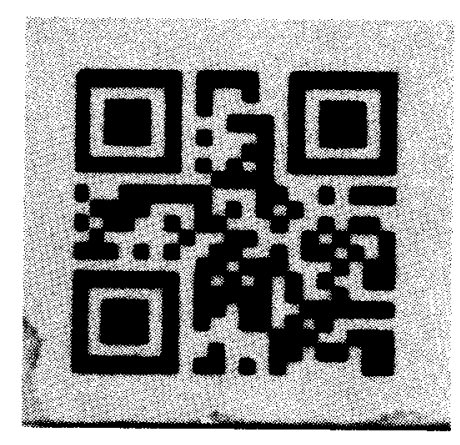

Fig. 4. $\mathrm{QR}$ code $^{\circledR}$ drawn in the optically addressive thermal rewritable paper.

\section{References}

1. K. Tsutsui, T. Yamaguchi, and K. Sato, Jpn. J. Appl. Phys., 33 (1994) 5925.

2. M. Hosoda, T. Wada, T. Yamamoto, A. Kaneko, A. F. Garito and H. Sasabe, Jpn. J. Appl. Phys., 31 (1992) 1071.

3. C. R. Fincher Jr., M. Ozaki, M. Tanaka, D. L. Peebles, L Lauchiau, S. J. Heeger and A. G. MacDiamid, Phys. Rev., B20. (1979) 1589.

4. M. Wan, T. Wada and H. Sasabe, Thin Solid Films, 208 (1992) 1.

5. M. Tian, T. Wada, L. Wang and H. Sasabe, Nonlinear Optics, 15 (1996) 205. 